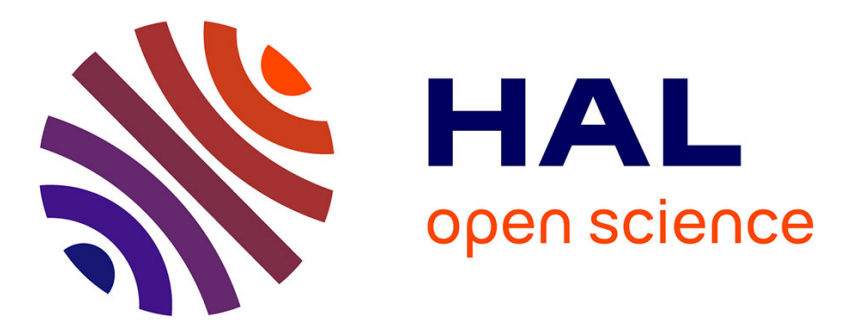

\title{
HDLs induce raft domain vanishing in heterogeneous giant vesicles
}

Nicolas Puff, Antonin Lamazière, Michel Seigneuret, Germain Trugnan, Miglena I. Angelova

\section{To cite this version:}

Nicolas Puff, Antonin Lamazière, Michel Seigneuret, Germain Trugnan, Miglena I. Angelova. HDLs induce raft domain vanishing in heterogeneous giant vesicles. Chemistry and Physics of Lipids, 2005, 133 (2), pp.195-202. 10.1016/j.chemphyslip.2004.10.003 . hal-02443635

\section{HAL Id: hal-02443635 \\ https://hal.sorbonne-universite.fr/hal-02443635}

Submitted on 17 Jan 2020

HAL is a multi-disciplinary open access archive for the deposit and dissemination of scientific research documents, whether they are published or not. The documents may come from teaching and research institutions in France or abroad, or from public or private research centers.
L'archive ouverte pluridisciplinaire HAL, est destinée au dépôt et à la diffusion de documents scientifiques de niveau recherche, publiés ou non, émanant des établissements d'enseignement et de recherche français ou étrangers, des laboratoires publics ou privés.

\section{(1) (1) $\$$}

Distributed under a Creative Commons Attribution - NonCommercial - NoDerivatives| 4.0 


\section{HDLs induce raft domain vanishing in heterogeneous giant vesicles}

Nicolas Puff ${ }^{*}$, Antonin Lamazière ${ }^{\#}$, Michel Seigneuret ${ }^{\&}$, Germain Trugnan\#, and Miglena I. Angelova ${ }^{*}$

* Laboratoire de Physicochimie Biomoléculaire et Cellulaire, Université Pierre et Marie Curie, CNRS UMR 7033, Case 138, 4 Place Jussieu, 75252 Paris Cedex 05, France;

\# INSERM U538, CHU St Antoine, 27 rue Chaligny, 75012 Paris, France;

\& Institut Cochin, U567-UMR8104, Département de Biologie Cellulaire, 22 rue Méchain, 75014, France.

Corresponding author:

Miglena I. Angelova

Present address: INSERM U538, CHU St Antoine, 27 rue Chaligny, 75012 Paris, France. Fax: +33 (0)140011390; e-mail : angelova@ccr.jussieu.fr 


\begin{abstract}
Cholesterol efflux from the plasma membrane to HDLs is essential for cell cholesterol homeostasis. Recently, cholesterol-enriched ordered membrane domains, i.e. lipid rafts have been proposed to play an important role in this process. Here we introduce a new method to investigate the role of HDL interactions with the raft lipid phase and to directly visualize the effects of HDL-induced cholesterol efflux on rafts in model membranes. Addition of HDLs to giant lipid vesicles containing raft-type domains promoted decrease in size and disappearance of such domains as visualized by fluorescence microscopy. This was interpreted as resulting from cholesterol efflux from the vesicles to the HDLs. The raft vanishing rate was directly related to the HDL concentration. Evidence for a direct interaction of HDLs with the membrane was obtained by observing mutual adhesion of vesicles. It is suggested that the present method can be used to study the selective role of the bilayer lipid phase (raft and non-raft) in cholesterol efflux and membrane-HDL interaction and their underlying mechanisms. Such mechanisms may contribute to cholesterol efflux in vivo.
\end{abstract}

Keywords: HDL, lipoproteins, GUV, lipid raft, cholesterol homeostasis, adhesion

Abbreviations: GUV, giant unilamellar vesicle; PC, Egg yolk L- $\alpha$-phosphtidylcholine; SM, egg yolk sphingomyelin; Chol, cholesterol; DiQ, 4-(p-(dihexadecylamino)styryl)-Nmethylquinolinium iodide. 


\section{Introduction}

High density lipoproteins play a pivotal role in cholesterol efflux from cell, a process which is essential to cellular cholesterol homeostasis and represents the first step of reverse cholesterol transport. There appear to be two distinct mechanisms involved in cellular cholesterol efflux : (1) an active efflux by which apo-A1, the main protein component of HDL gets lipidated, yielding pre-beta HDL ; (2) a passive efflux involving diffusion of cholesterol from the plasma membrane to HDLs along its concentration gradient (for reviews, see Yokoyama, 1998, Fielding and Fielding, 2001). The molecular mechanisms by which cholesterol is transferred from the membrane to HDLs or their precursors in these two processes remain largely unknown. In particular, the respective roles of the interactions of HDLs with specific plasma membrane proteins and with the plasma membrane lipid matrix is not well understood. Although active cholesterol transport to apo-A1 appears to involve the activity of the ATP-binding cassette transporter A1, no direct interaction between these two proteins has been demonstrated. Oppositely, while the passive cholesterol efflux to HDL can in principle occur directly from the lipid matrix, a role for the scavenger receptor SR-B1 in this process is also suggested. Another related question is the possible role of specific microdomains, such as rafts or caveolae, in cholesterol efflux to HDL. Such cholesterol and sphingolipid-enriched ordered microdomains are thought to exist in plasma membranes where they recruit specific proteins and may represent a dynamic cholesterol pool (Simons and Ikonen, 2000). The rate of efflux from cholesterol rich domains was initially predicted to be slow (Bielicki et al., 1992). However selective efflux of cholesterol from caveolae or from defined types of lipid rafts either to apo-A1 or HDLs has also been observed (Mendez et al., 2001, Sviridov et al., 2002, Drobnik, 2002). Both ABCA1 (Drobnik, 2002) and SR-B1 (Peng et al., 2004) have been reported to selectively localize in rafts or caveolae. However, also in this case, the relative role of raft proteins or of the raft lipid phase is difficult to evaluate. For example, it is not known whether HDLs and their precursors have a specific affinity for the raft lipid phase. 
An efficient approach to evaluate the specific role of lipids in membrane-associated processes is the use of artificial lipid vesicles. Indeed, both small and large unilamellar vesicles have been extensively used for the study of membrane-HDL cholesterol exchange and have yielded important results (Phillips et al., 1987; Toledo et al., 2000). It is nevertheless still a matter of debate in these studies whether such exchange occurs through cholesterol aqueous diffusion, HDL-membrane collisions or HDL-membrane direct interactions (see discussion in Steck et al., 1988). A limitation is that SUVs and LUVs, due to their small size, can only partially mimic cell membranes. For example, raft domain -related phenomena cannot be directly studied with such vesicles. On the other hand, the more recently introduced giant unilamellar vesicles constitute a model system size wise more representative of cell membranes (Angelova and Dimitrov, 1986; Menger and Angelova, 1998). An additional benefit is that many processes involving GUVs can be studied by optical microscopy. GUVs can be prepared by electroformation over a wide range of lipid compositions. Recently, several authors have used such electroformed GUVs containing cholesterol and sphingolipids to visualize raft-like (liquid ordered, lo, phase) microdomains and to study biologically relevant phenomena (Dietrich et al., 2001; Staneva et al., 2004; Bacia et al., 2004).

In this work, we show that raft-GUVs can be used to study HDL - membrane interactions. In particular, we present direct optical microscopy observation of raft-type domains vanishing due to heterogeneous vesicle interaction with human blood HDLs.

\section{Materials and methods}

\subsection{Reagents}

EYPC (egg yolk phosphatidylcholine), EYSM (egg yolk sphingomyelin) and cholesterol were purchased from Sigma. The lipophilic membrane probe Di-Q (4-(p-(dihexadecylamino)styryl)N-methylquinolinium iodide) (Ex/Em : 562/600+) was obtained from Molecular Probes. 
Human HDLs were purified from healthy, clinically stable volunteers with no lipid modifying medication, who gave informed consent, after approval by the Service de Biochimie B, Hôpital St-Antoine (Paris, France).

\subsection{Giant unilamellar vesicles (GUVs)}

GUVs were prepared by electroformation (Angelova and Dimitrov, 1986 ; Angelova, 2000), with $\mathrm{PC} / \mathrm{SM} / \mathrm{Chol} 60: 20: 20 \mathrm{~mol} / \mathrm{mol}$ or $\mathrm{PC} / \mathrm{DiQ} / \mathrm{SM} /$ Chol $55: 5: 20: 20 \mathrm{~mol} / \mathrm{mol}$ (unless otherwise indicated). DiQ was chosen for its good capacity to resist to photobleaching compare to other probe used in the past (for example fluorescent lipid analogue NBD-PC). The electroformation protocol was similar to Staneva et al. 2004. Briefly, the lipid mixture was prepared in solvent, diethyl ether/methanol/chloroform 70:10:20 v/v, at $0.4 \mathrm{mg} / \mathrm{ml}$ total lipid. A droplet of lipid solution (approximately $1 \mu \mathrm{l}$ ) was deposited (avoiding sliding) on each of two parallel platinium wires (diameter $0.8 \mathrm{~mm}$, distance between axes $3 \mathrm{~mm}$ ) and dried under a gentle steam of nitrogen for $30 \mathrm{~min}$. An alternating current $(A C)$ electrical field $(10 \mathrm{~Hz}$ and $100 \mathrm{mV}$ ) was applied to the electrodes at room temperature $\left(25^{\circ} \mathrm{C}\right)$. A thermocouple positioned at a distance of about $0.5 \mathrm{~mm}$ from the place of observation was used to monitor the temperature during the experiment. The dried lipid film was hydrated by adding $1.5 \mathrm{ml}$ of a low ionic strength buffer (HEPES : $0.5 \mathrm{mM}, \mathrm{pH} 7.4$, conductivity $\sigma=20 \mu \mathrm{S} / \mathrm{cm}$ ). The temperature and the applied voltage were gradually increased (during $10 \mathrm{~min}$ ) up to respectively $30^{\circ} \mathrm{C}$ and $200 \mathrm{mV}$ pp. The voltage was increased during $2 \mathrm{~h}$ up to $400 \mathrm{mV}$ to promote electroformation. Using this protocol, at least 10 GUVs of diameters $40-90 \mu \mathrm{m}$ were present in each preparation.

\subsection{Isolation of HDLs}

HDLs $(1,063<$ density, $\mathrm{d}<1.21 \mathrm{~g} / \mathrm{ml})$ were isolated from human plasma by sequential flotation ultracentrifugation as described by Benlian et al. (2000). Briefly, serum was 
separated from other blood components and its density was adjusted to $d=1.063 \mathrm{~g} / \mathrm{ml}$ with solid potassium bromide. EDTA ( $3 \mathrm{mM})$ was then added to prevent aggregation of lipoprotein complexes. The preparation was first centrifuged for $5 \mathrm{~h}$ at $100000 \mathrm{rpm}$ in a TLA 100.4 rotor (Beckman Instruments) at $4^{\circ} \mathrm{C}$ in order to remove $\mathrm{LDL}$ and VLDL $(\mathrm{d}<1.063 \mathrm{~g} / \mathrm{ml})$ from the intranatants containing HDLs and other serum proteins. The density was readjusted at 1.21 $\mathrm{g} / \mathrm{ml}$ with potassium bromide, and after a second centrifugation ( $\left.6 \mathrm{~h}, 100000 \mathrm{rpm}, 4^{\circ} \mathrm{C}\right) \mathrm{HDLs}$ were collected as the top layer and dialyzed for $12 \mathrm{~h}$ against a solution of PBS and EDTA (3 $\mathrm{mM})$ at $4^{\circ} \mathrm{C}$.

\subsection{Characterization of HDLs}

The concentration of apolipoproteins in the HDL preparations was determined by immunonephelemetry using a laser Nephelometer analyzer BN II (Behring Instruments) and using specific antibodies ( $\mathrm{N}$ antisera to Apo $\mathrm{A}-\mathrm{I}$ and Apo $\mathrm{B}$ ). The apolipoprotein concentrations are proportional to the intensities of the scattered light from their antibody complexes and were quantified by comparison with a standard. This confirmed the absence of Apo B in the sample. The cholesterol and triglyceride concentration were also measured using the Synchron CX4CE system (Beckman Instrument) to confirm the purity of our separation.

The purity of the HDL preparations was also checked by SDS gel electrophoresis and agarose gel chromatography with total cholesterol detection. SDS gel electrophoresis showed the presence of apoA1 and apoA2 as well as of a minor serum albumin band. The latter amounted to less than $2 \%$ of total. Agarose gel chromatography followed by total cholesterol detection showed no detectable trace of LDL in our HDL preparation. 


\subsection{Addition of HDLs}

The bulk addition of HDLs to electroformed GUVs was carried out with a pipetman (Gilson: $200 \mu \mathrm{l})$ or a Hamilton microsyringe $(200 \mu \mathrm{l})$. The addition has to be performed drop by drop at the surface of the chamber and as far as possible from the electrodes to avoid GUV collapse or disturbance by mechanical flux or osmotic chock. Added volumes ranged from $200 \mu \mathrm{l}$ to $400 \mu \mathrm{l}$ of an HDL solution of $2 \mathrm{mg} / \mathrm{ml}$ in apo-A1. This corresponds to final bulk HDL concentration of 0.1 to $0.2 \mathrm{mg} / \mathrm{ml}$ in Apo A-I. All observations presented below are based on at least five experiments of the same kind.

\subsection{Video microscopy}

A Zeiss Axiovert 200 microscope, equipped with a lambda 10-2 (Sutter Instrument Co.), plus a CCD camera (Cool SNAP HQ), and a fluorescent unit (fluo arc N HBO 103, Zeiss) was used for GUV imaging. The experiments were computer-controlled using the Metamorph software (Roper Scientific). The morphological transformations and dynamics of the heterogeneous GUV membranes were followed by phase contrast microscopy and in fluorescence using the Zeiss filter set $15(E x / E m=546 / 590+)$.

\section{Results}

In this study, in order to monitor cholesterol exchange from all physiological HDL classes, we have used unfractionated HDLs from human plasma. The electroformed GUVs used to monitor the effect of HDLs on lipid raft-type (lo phase) domains were composed of PC, SM and cholesterol (PC/SM/Chol 60:20:20 mol/mol). Figure 1a illustrates the typical appearance of liquid-ordered domains thought to represent lipid rafts on such vesicles as visualized by 
fluorescence microscopy using the fluorescent probe DiQ. As already described, this probe is excluded from the more ordered lipid phase (lo phase) which, under the conditions of figure 1a (PC/DiQ/SM/Chol 55:5:20:20, $31^{\circ} \mathrm{C}$ ) appears as a dark round shaped domain within the bright Id liquid disordered phase. During the time scale of the experiment, the liquid-ordered domains undergo visible lateral diffusion (not shown). There are occasionally several initial liquid-ordered domains per vesicles ( 2 or 3 at $30^{\circ} \mathrm{C}$ ) which ultimately coalesce as a result of lateral diffusion thereby decreasing line tension energy. Figures $\mathbf{1 b}-\mathbf{l}$ show the evolution of the liquid-ordered domains -containing vesicle following a bulk injection of plasma HDLs at a final concentration in Apo-Al of $0.1 \mathrm{mg} / \mathrm{ml}$. A continuous decrease of the diameter of the liquid-ordered domains occurs (simultaneously with the domain lateral diffusion) and leads to its disappearance in ca. $30 \mathrm{~min}$. This is likely to be due to the progressive decrease of the cholesterol content of the GUV bilayer consecutive to its efflux towards the HDL along its concentration gradient. It was established (de Almeida et al., 2003) in similar lipid mixtures that for the PC/SM ratio and at the temperature of Fig. 1, a molar fraction of at least $10 \%$ of cholesterol is necessary to observe the lo phase liquid-ordered domains. It is therefore likely that upon the addition of HDLs the cholesterol content of the vesicle is lowered below this value in a timescale of 30 minutes. On the other hand at this timescale, no decrease of the vesicle diameter was observed within our experimental resolution (about $5 \%$ in surface). The later observation suggests that phospholipid efflux from GUVs to HDL was limited or not significant. This is consistent with the fact that lipid-free or lipid poor Apo-A1, which are the main potential phospholipid acceptors make less than $5-10 \%$ of circulating HDLs. The fact that cholesterol depletion of the vesicle does not lead to vesicle size decrease may be due to the well-documented condensing effect of cholesterol on phospholipids, i.e. the decrease in area due to cholesterol removal is compensated by the increase in phospholipid mean molecular area. Furthermore, due to phase contrast microscopy and digital resolution limits, a GUV surface decrease of less than $5 \%$ would not be detectable.

Similar results were obtained in 5 distinct experiments. Although the liquid-ordered domain decrease was each time followed on a single vesicle, the whole vesicle population was 
routinely observed at a lower magnification at the beginning and at the end of each experiment. It was found that $90 \%$ of the vesicles initially contained liquid-ordered domains but ultimately became devoid of such domains. The cholesterol efflux therefore appears to be a general process involving all vesicles. Fig. 2 shows that the kinetics of liquid-ordered domains size decrease is linear (at least during the time frame in which such size can be measured). Similar experiments were performed at two different HDL concentrations. As shown in Fig. 2, increasing HDL concentration $(\boldsymbol{\Delta})$ leads to faster liquid-ordered domains vanishing.

Apart from the continuous disappearance of the liquid-ordered domains domain due to cholesterol efflux from the bilayer, addition of HDL was found to have another effect on GUVs. In the presence of HDL, the adjacent vesicles underwent mutual strong and stable adhesion, as visualized in phase contrast, Fig. 3a-d, and in fluorescence, Fig. $3 \mathbf{a}^{*}-\mathbf{d}^{*}$. In the absence of HDL, such adhesion was never observed. It is likely that such adhesion is due to interaction of specific HDL species with the vesicle membrane that leads to cross linking. Interestingly, such adhesion was detected with GUVs, with or without cholesterol, and therefore with or without the liquid ordered phase, suggesting that the presence of such a phase is not a prerequisite for the HDL-lipid bilayer interaction. On the other hand, our experiments show that the presence of specific proteins in the lipid membrane is not required for its direct interaction with the HDLs to take place.

\section{Discussion}

In the present works, we have introduced a new method for studying of HDL-membrane interactions and of HDL-induced cholesterol efflux. The benefit of artificial vesicles for the study of membrane-associated process is their modularity and controllability which allows one to study the effect of compositional and physico-chemical parameters. In this framework, the obvious advantage of GUVs is that these are closer in size and curvature to plasma membranes and can be used to mimic several aspects of the lateral heterogeneity found in 
situ. While one study of HDL-GUV interaction has already appeared (Tricerri et al., 2002), this was done on homogenous membranes in the absence of cholesterol. Here, we have taken advantage of the ability of GUV to accommodate raft-type domains. The cholesterol and sphingomyelin-enriched domains that can be visualized by optical microscopy on GUVs are probably larger than biological rafts. Furthermore, direct evidence that biological rafts correspond to a liquid-ordered phase are limited. However, several studies suggest that these domains can be considered as partially representative of the behavior of rafts in situ. Indeed, several biological processes involving lipid rafts have been mimicked with liquidordered domains-containing GUVs, including glycosphingolipid partition (Dietrich et al., 2001), cholera toxin binding (Bacia et al. 2004a) and intrinsic proteins (Bacia et al. 2004b) and membrane budding phenomena (Staneva et al. 2004). Here, we have been able to directly visualize an effect which may be relevant to the role of rafts in HDL-induced cellular efflux, namely the selective disappearance of raft domains in GUVs in the presence of HDLs. This can be related to several studies that have reported a selective efflux of cholesterol from caveolae or rafts either to HDLs. In this instance, our study raises one question which is relevant for both the in situ and the GUV situation: do such effects reflect a direct and selective extraction of cholesterol from the raft domain or a re-equilibration of the lipid phase following a non selective removal of cholesterol. GUVs may provide an adequate system to document this question. A direct perturbation of the raft phase due to a disturbing effect of HDL interaction with lipid might also contribute to the decrease of the raft domains.

It is interesting to note that cholesterol depletion occurs from GUVs in spite of the fact that these are attached to the electrode. The electrode is covered with lipid layers that might in principle function as a reservoir and replenish cholesterol extracted from GUVs. The fact that this does not occur indicate that either cholesterol is also extracted from electrode lipid layers or that there is a diffusion barrier between the electrode and GUV lipid phases which significantly slows down lipid lateral diffusion between these.

Our experiments also underline several other questions that are pivotal for the understanding of the mechanism of HDL induced cholesterol efflux. For example the mechanism by which 
cholesterol is extracted from lipid bilayers has been a matter of debate. The observation of situation in which the rate of exchange is independent of acceptor concentration has been invoked in favor of an aqueous diffusion of cholesterol monomers (Phillips et al. 1987). Here we find that the rate of raft disappearance from GUVs is dependant on HDL concentrations. As stressed by Steck et al., (1988), such as situation may occur in an aqueous diffusion mechanism when the acceptor is not in excess but may be also representative of other mechanisms such as collision, activation-collision or direct interaction. The possibility of an efflux mechanism involving bilayer-HDL interactions is particularly appealing in our case since we were able to obtain evidence for such interaction by observing HDL-induced adhesion of GUVs. This raises the biologically important question of whether HDLs interact selectively with the raft or the non raft phase. GUV studies with fluorescent HDLs may help documenting this aspect. A related question is which HDL species are involved. Previous studies suggest that pre-beta or "pre beta-like » HDLs are more prone to interact with membranes and to promote cholesterol efflux. Future studies with fractionated or reconstituted HDLs will be used to investigate this question.

In conclusion, we show here that the use of GUVs provide a new method to study the role of the membrane lipid phase and of lipid rafts in HDL-induced cholesterol exchange. This provides a new approach to evaluate the relative contributions of receptor-associated processes and of specific or non-specific membrane lipid-HDL interactions in cellular cholesterol efflux.

\section{Acknowledgements}

We thank Pascale Benlian for introducing us to HDL preparation methods and Alexandre Hamez for technical support. The CNRS/MR joint programme «Dynamics and Reactivity of Biological Assemblies» supported this work. 


\section{References}

Almeida, R., Fedorov, A., Prieto, M., 2003. Sphingomyelin/Phosphotidylcholine/ Cholesterol phase diagram : boundaries and composition of lipid raft. Biophys. J., 85, 2406-2416.

Angelova, M.I., 2000. Liposome electroformation. In: Luisi, P.L., Walde, P. (Eds.), Giant Vesicles, Perspectives in Supramolecular Chemistry, vol. 6. Wiley, Chichester, 27-36.

Angelova, M.I. and Tsoneva, I., 1999. Interactions of DNA with giant liposomes. Chem. Phys. Lipids 101, 123-137

Angelova, M. and Dimitrov, D., 1986. Liposome electroformation. Faraday Discuss. Chem. Soc. $81,303-311$.

Bacia, K., Scherfeld, D., Kahya, N., Schwille, P., 2004a. Fluorescence correlation spectroscopy relates rafts in model and native membranes. Biophys. J. 87, 1034-1043.

Bacia, K., Schuette, C. G., Kahya, N., Jahn, R., and Schwille P., 2004b. SNAREs prefer liquid-desordered over "raft"- (liquid-ordered) domains when reconstituted into Giant Unilamellar Vesicles. J. Biol. Chem. 279, 37951-37955.

Benlian, P., Cansier, C., Hennache, G., Khallouf, O., Bayer, P., Duron, F., Carrat, F., Couderc, R., Chazouilleres, O., Bardet, J., Bouchard, P., Poupon, R., Masliah, J., Bereziat, G., 2000. Comparison of a new method for the direct and simultaneous assessment of LDL- and HDL-cholesterol with ultracentrifugation and established methods. Clin Chem., 46, 493-505.

Bielicki, J.K., Johnson, W.J., Weinberg, R.B., Glick, J.M., and Rothblat, G.H., 1992. Efflux of lipid from fibroblasts to apolipoproteins: dependence on elevated levels of cellular unesterified cholesterol. J. Lipid Res., 33, 1699 - 1709.

Corsico, B., Toledo, J., Garda, H., 2001. Evidence for a central Apolipoprotein A-I domain loosely bound to lipids in discoidal lipoprotein that is capable of penetring the bilayer of phospholipids vesicles, J. Biol. chem., 276, 16978 - 16985. 
Dietrich, C., Bagatolli, L., Volovyk, Z., Tompson, N., Levi, M., Jacobson, K. and Gratton, E., 2001. Lipid rafts reconstituted in model membranes. Biophys. J. 80, 1417-1428.

Drobnik W, Borsukova H, Bottcher A, Pfeiffer A, Liebisch G, Schutz GJ, Schindler H, Schmitz G., 2002. Apo Al/ABCA1-dependent and HDL3-mediated lipid efflux from compositionally distinct cholesterol-based microdomains. Traffic 3, 268-278.

Fielding, C.J., Fielding, P.E., 2001. Cellular cholesterol efflux. Biochim. Biophys. Acta, 1533, 175-189.

Ikonen, E., 2001. Roles of lipid rafts in membrane transport. Curr. Opin. Cell Biol. 13, 470477.

Mendez, A.J., Lin, G., Wade, D.P., Lawn, R.M., Oram J.F. 2001. Membrane Lipid Domains Distinct from Cholesterol/Sphingomyelin-Rich Rafts Are Involved in the ABCA1mediated Lipid Secretory Pathway. J. Biol. Chem., 276, 3158-3166.

Menger, F.M., Angelova M.I. 1998. Giant vesicles: Imitating the citological processes of cell membranes.Acc. Chem. Res., 31, 789-797.

Peng, Y., Akmentin, W., Connelly, M.A., Lund-Katz, S., Phillips, M.C., Williams, D.L., 2004. Scavenger Receptor BI (SR-BI) Clustered on Microvillar Extensions Suggests that This Plasma Membrane Domain Is a Way Station for Cholesterol Trafficking between Cells and High-Density Lipoprotein. Mol. Biol. Cell, 15, 384-396.

Phillips, M.C., Johnson, W.J. and Rothblat, G. H., 1987.Mechanisms and consequences of cellular cholesterol exchange and transfer. Biochim Biophys Acta, 906, 223-276.

Simons, K. and Ikonen, E., 1997. Functional rafts in cell membranes. Nature 387, 569-572.

Simons, K., and Ikonen, E., 2000. How cell handle cholesterol. Science 290, 1721-1726.

Staneva, G., Angelova, M., and Koumanov, K. 2004. Phospholipase $A_{2}$ promotes raft budding and fission from giant liposomes. Chem. Phys. Lipids, Volume 129, Issue 1, $53-62$.

Steck, T.L., Kezdy, F.J., and Lange Y., 1988. An activation-collision mechanism for cholesterol transfer between membranes. J. Biol. Chem., 263, 13023-13031. 
Sviridov, D., Fidge, N., Beaumier-Gallon, G. and Fielding, C., 2001. Apolipoprotein A-I stimulates the transport of intracellular cholesterol to cell-surface cholesterol-rich domains (caveolae). Biochem. J., 58, 79-86.

Toledo, J. D., Tricerri, A., Córsico, B., Garda, H.A., 2000. Cholesterol Flux between Lipid Vesicles and Apolipoprotein AI Discs of Variable Size and Composition. Arch. Biochem. and Biophys., 380, Issue 1, 63-70.

Tricerri, A., Sanchez, S., Arnulphi, C., Durbin, D., Gratton, E., Jonas, A., 2002. Interaction of apolipoprotein A-I in three different conformations with palmitoyl oleoyl phosphatidylcholine vesicles. J. Lipid Res., 43, 187-197.

Yokoyama, S., 2000. Release of cellular cholesterol: molecular mechanism for cholesterol homeostasis in cells and in the body. Biochim. Biophys. Acta, 1529, 231-244. 


\section{Figure captions}

Figure 1. Evolution of the raft-containing vesicle (PC/DiQ/SM/Chol 55:5:20:20, $31^{\circ} \mathrm{C}$ ) following a bulk injection of plasma HDLs (HDLs bulk concentration $0.1 \mathrm{mg} / \mathrm{ml}$ in apo-A1). (a) Initial raft-GUV in fluorescence (one raft appears as a dark round shaped domain); (b-I) Continuous decrease of the diameter of the raft-like (lo phase) domain. Bar $=20 \mu \mathrm{m}$.

Figure 2. Kinetics of raft size decrease: relative raft surface as a function of time for different HDL final bulk concentrations, in apo-A1: $0.1 \mathrm{mg} / \mathrm{ml}(\bullet)$, and $0.2 \mathrm{mg} / \mathrm{ml}(\boldsymbol{\Delta})$.

Figure 3. Strong and stable adhesion between two GUVs (PC/DiQ/SM/Chol 55:5:20:20, $31^{\circ} \mathrm{C}$ ) following the bulk addition of human plasma HDLs (bulk concentration of $0.1 \mathrm{mg} / \mathrm{ml}$ in apo-A1) ; (a-d) in phase contrast; $\left(a^{*}-d^{*}\right)$ in fluorescence. Bar $=20 \mu \mathrm{m}$. 


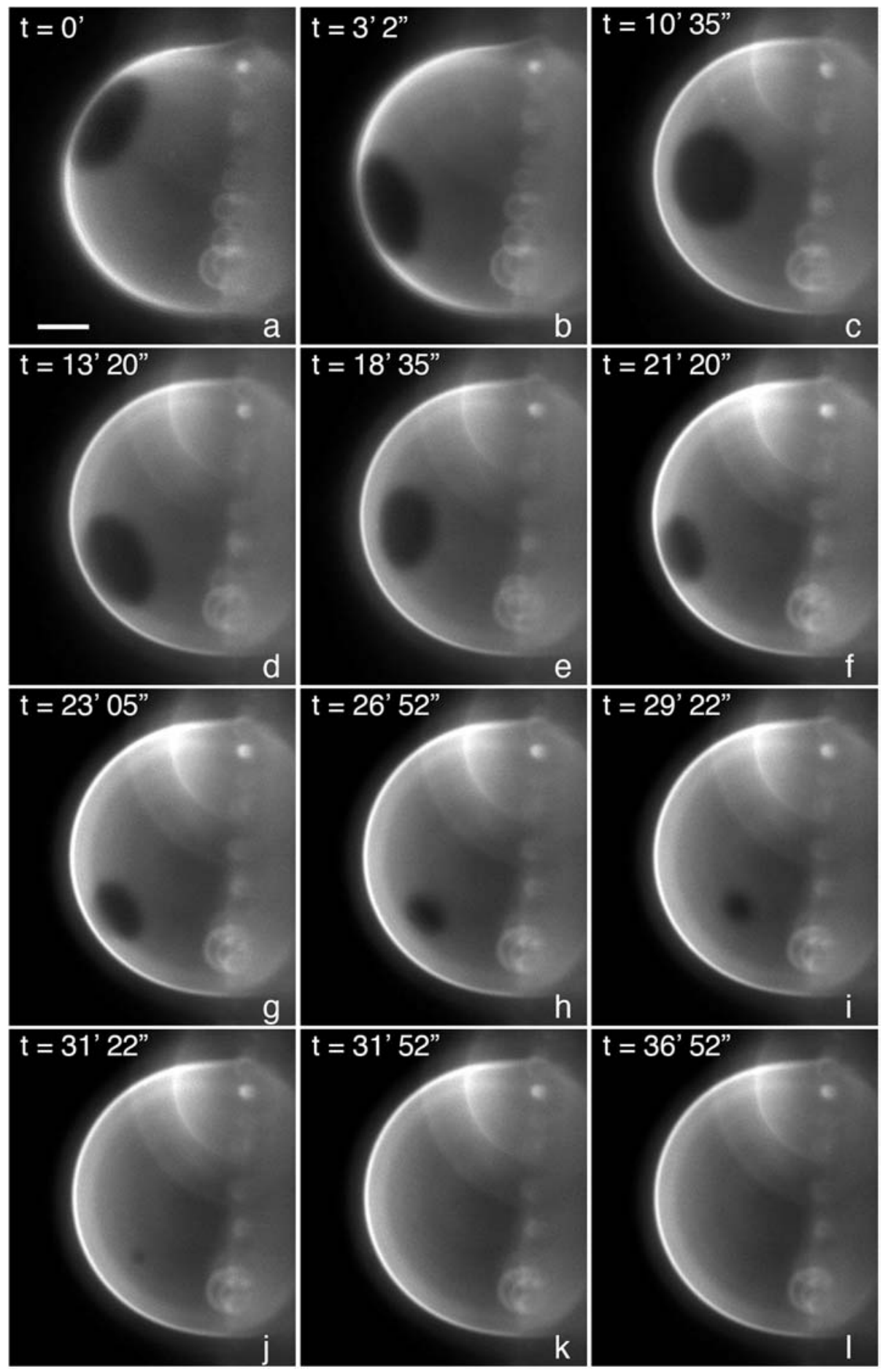

Figure 1 


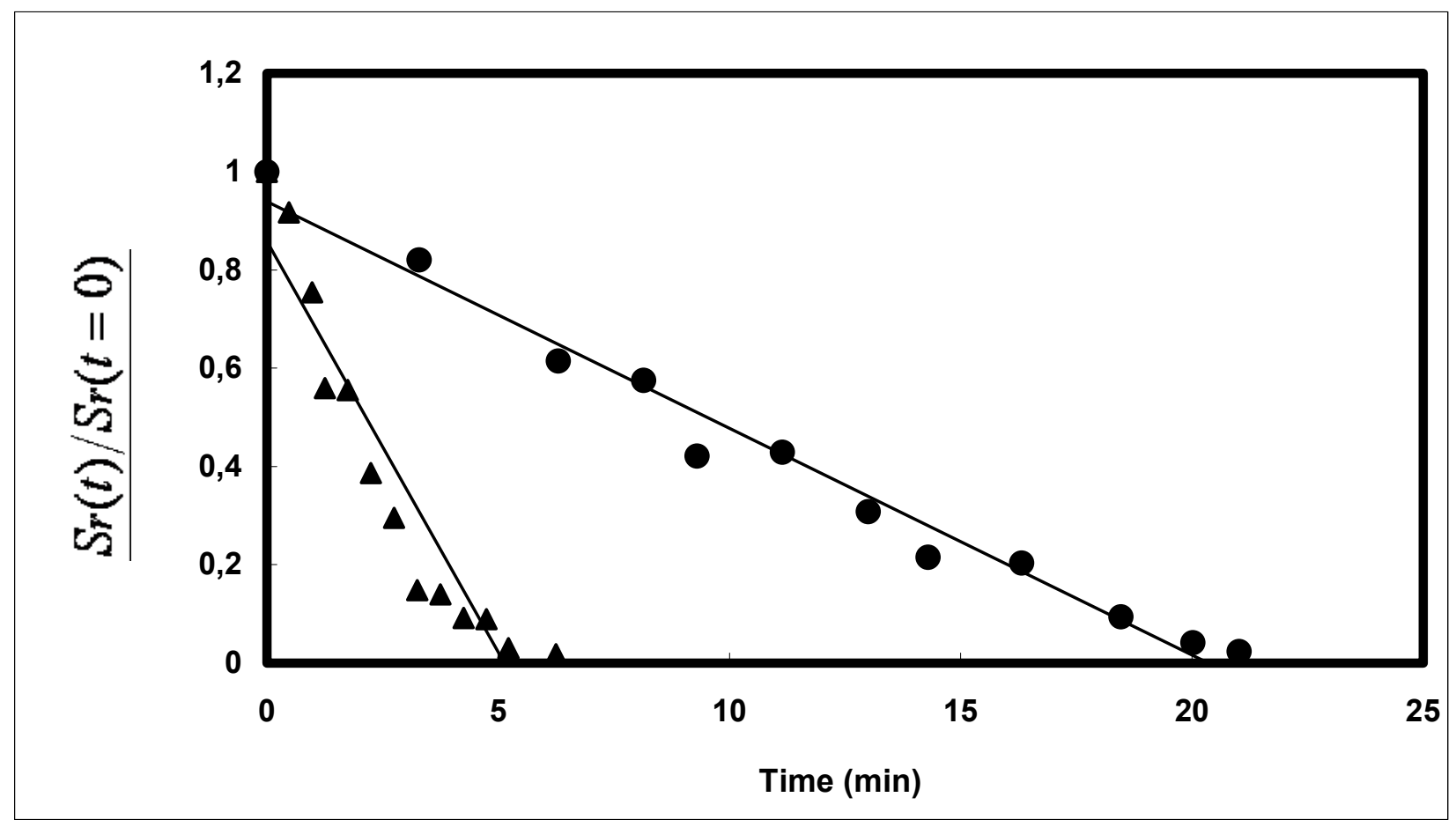

Figure 2 

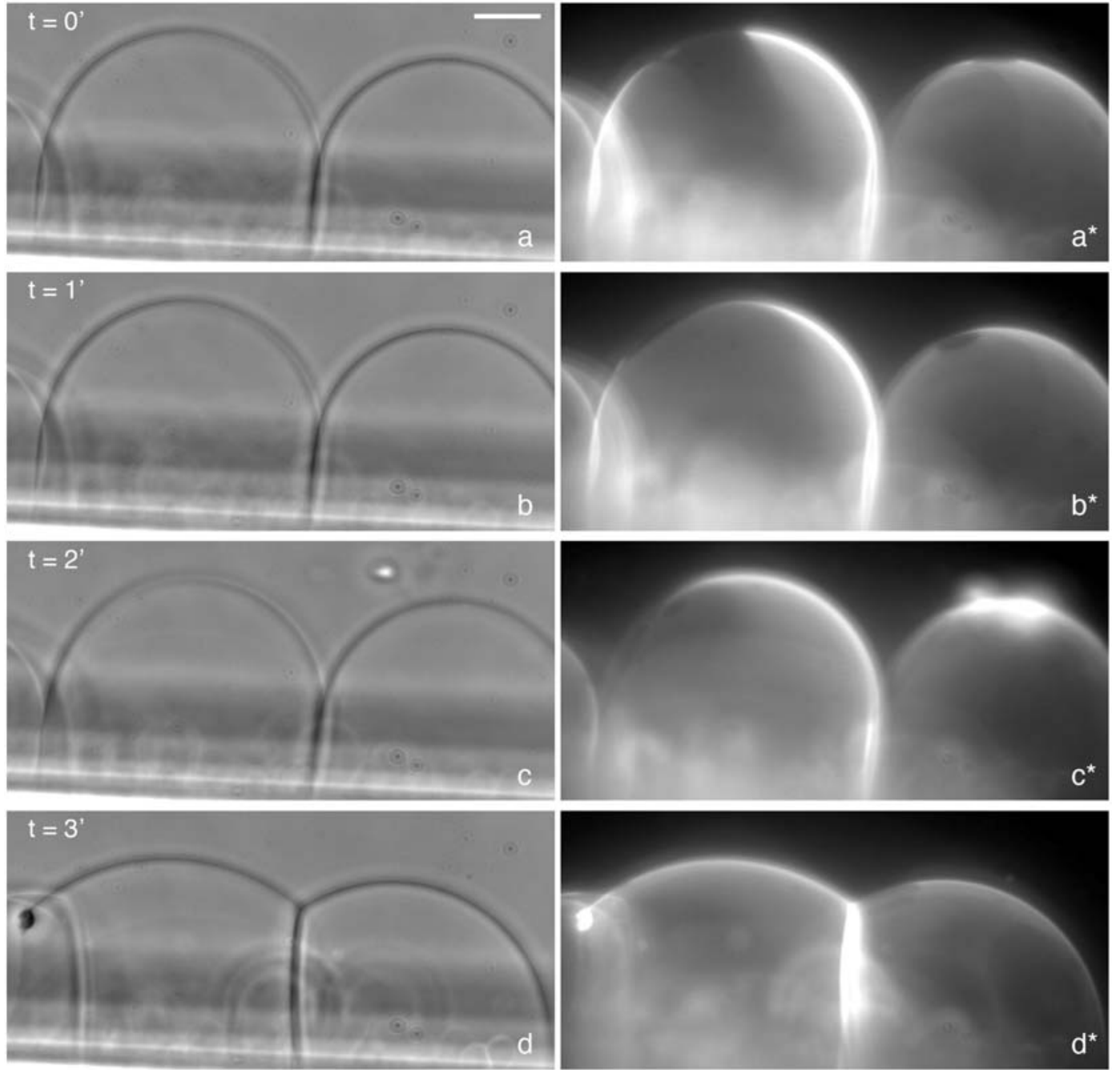

Figure 3 